\title{
Characterization of a Layered Lithium Manganese-rich Oxide Cathode Material via Scanning Transmission Electron Microscopy
}

\author{
Aaron C. Johnston-Peck, Leonid A. Bendersky and Andrew A. Herzing
}

Material Measurement Laboratory, National Institute of Standards and Technology, Gaithersburg, MD, 20899, USA

Energy storage technologies, including batteries, are a critical component of the transformation of the energy economy toward renewable sources and reduced dependency on fossil fuels. Energy storage technologies are a potential route to buffer wind and solar power's intermittent electricity generation. Storage systems will supply the necessary electricity to supplement these renewable sources and match demand during conditions where solar or wind power generation is impaired. Batteries can provide the necessary storage, however, to become feasible, improvement to energy densities, lifetime, and stability need to be made in parallel with reductions in production costs. To design and produce these next generation batteries, it is necessary to develop a comprehensive understanding of the structure and electrochemical processes of the materials that are to be utilized.

Layer compounds such as $\mathrm{Li}_{2} \mathrm{MnO}_{3}$ have high capacities in excess of $200 \mathrm{mAh} / \mathrm{g}$ [1]. The $\mathrm{Li}_{2} \mathrm{MnO}_{3}$ structure is comprised of alternating layers of lithium atoms and lithium with manganese atoms (or other transition metals) in an ordered sequence of Li-M-M-Li-M-M-Li separated by layers of oxygen atoms. In this on-going study, a layered cathode material containing cobalt and nickel in addition to manganese was characterized by transmission electron microscopy and aberration corrected scanning transmission electron microscopy.

STEM images of the as-synthesized particles (Figure 1) reveal a disc- or plate-like morphology with well-defined surface facets. Atomic resolution imaging and electron diffraction indicate the pristine particles are a solid solution in the monoclinic $\mathrm{C} 2 / m$ phase. No evidence was found to support the presence of long range ordered tetragonal $\mathrm{R} \overline{3} m$ phase, which has been proposed to coexist with the monoclinic phase in some of these materials [2]. However, at the surface of the particles a spinel phase was found. These regions appear brighter in the HAADF STEM images suggesting a higher average Z (atomic number) than the rest of the material. Energy dispersive X-ray spectroscopy (EDS) and electron energy loss spectroscopy (EELS) confirmed that this spinel phase is nickel-rich. Planar defects along the c axis were observed along with three crystal variants with orientations of [100], [110], and [1피 which result in the rotation of the lattice and the creation of domains. Further studies are planned for materials that have undergone charge/discharge cycling.

\section{References:}

[1] M.M. Thackeray et al, J. Mater. Chem. 15 (2005), p.2257.

[2] M.M. Thackeray et al, J. Mater. Chem. 17 (2007), p.3112.

[3]A.C.J.P. acknowledges support of a the National Research Council Postdoctoral Research Associateship Programs. 


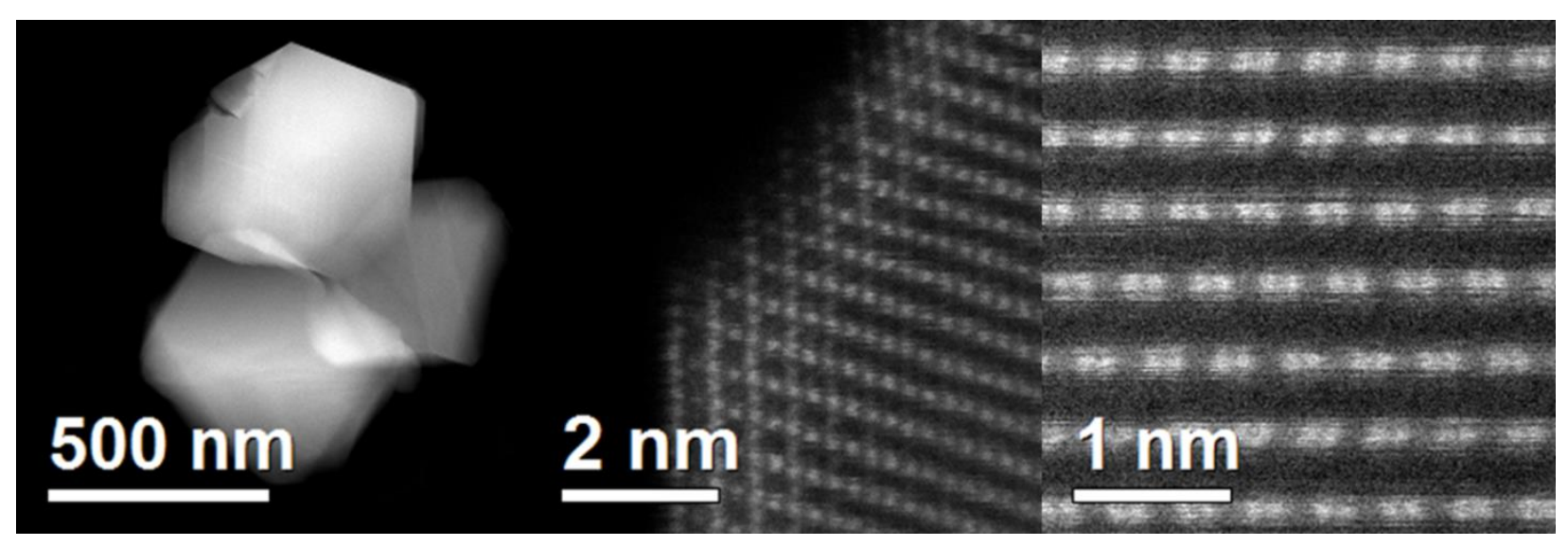

Figure 1. Annular dark field STEM images of the pristine $\mathrm{Li}_{2} \mathrm{TMO}_{3}$ sample, showing the general morphology of the particles, the formation of the Ni-rich spinel phase at the surface, and the presence of a two different crystal variants along the $\mathrm{c}$ axis. 